\title{
Erratum: Table of Contents, cover caption
}

\section{Nature Immunology 4, i (2003).}

In the cover caption of the December 2003 issue of Nature Immunology, the second sentence should read "This 2-day-old embryo illustrates how one can monitor multilineage hematopoiesis in living zebrafish after transplantation of transgenic donor marrow carrying red (eyrthocytes;

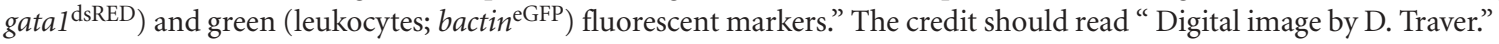

